\title{
Disturbed subjective sleep characteristics in adult patients with long-standing type 1 diabetes mellitus
}

\author{
M. van Dijk • E. Donga $\cdot$ J. G. van Dijk • \\ G. J. Lammers • K. W. van Kralingen • O. M. Dekkers • \\ E. P. M. Corssmit • J. A. Romijn
}

Received: 24 January 2011 / Accepted: 7 April 2011 /Published online: 15 May 2011

(C) The Author(s) 2011. This article is published with open access at Springerlink.com

\begin{abstract}
Aims/hypothesis Decreased sleep duration and/or impaired sleep quality negatively influence glucoregulation. The aim of this study was to assess subjective sleep characteristics in patients with type 1 diabetes, to relate sleep characteristics to long-term glycaemic control and to assess possible risk factors for impaired sleep.

Methods We studied 99 adult patients with type 1 diabetes (55 men, 44 women, duration of diabetes $26.9 \pm 1.2$ years) and 99 age-, sex- and BMI-matched non-diabetic controls. Subjective sleep characteristics were assessed by validated questionnaires, i.e. Pittsburgh Sleep Quality Index, Epworth Sleepiness Scale and the
\end{abstract}

M. van Dijk and E. Donga contributed equally to this study.

M. van Dijk $(\bowtie) \cdot$ E. Donga $\cdot$ O. M. Dekkers •

E. P. M. Corssmit · J. A. Romijn

Department of Endocrinology and Metabolic Diseases,

Leiden University Medical Centre,

PO Box 9600, 2300 RC Leiden, the Netherlands

e-mail: mvandijk2@lumc.nl

J. G. van Dijk · G. J. Lammers

Department of Neurology, Leiden University Medical Centre,

Leiden, the Netherlands

K. W. van Kralingen

Department of Pulmonology, Leiden University Medical Centre,

Leiden, the Netherlands

O. M. Dekkers

Department of Epidemiology, Leiden University Medical Centre, Leiden, the Netherlands

J. A. Romijn

Department of Internal Medicine, Academic Medical Centre,

Amsterdam, the Netherlands
Berlin Questionnaire. Glucoregulation was assessed by $\mathrm{HbA}_{1 \mathrm{c}}$ values. Clinical variables were obtained from medical charts. Depression was assessed by the Hospital Anxiety and Depression Scale (HADS). Peripheral polyneuropathy was assessed by neurological examination and quantitative sensory testing.

Results Of the patients with type 1 diabetes, $35 \%$ had subjective poor sleep quality compared with $20 \%$ of the control participants $(p=0.021)$. A higher proportion of the patients with type 1 diabetes were at increased risk for obstructive sleep apnoea (OSA) $(17.2 \%$ vs $5.1 \%, p=0.012)$. There was no significant association between individual sleep characteristics and $\mathrm{HbA}_{1 \mathrm{c}}$ values. On logistic regression analysis, the HADS depression score, presence of peripheral polyneuropathy, habitual snoring and other sleep disturbances (e.g. hypoglycaemia) were independently associated with poor sleep quality.

Conclusions/interpretation Adult patients with longstanding type 1 diabetes mellitus have disturbed subjective sleep quality and a higher risk for OSA compared with control participants. Subjective sleep disturbances are part of the complex syndrome of long-standing type 1 diabetes.

Keywords Glucoregulation · Sleep · Sleep disorders · Type 1 diabetes mellitus
Abbreviations
BQ Berlin Questionnaire
ESS Epworth Sleepiness Scale
HADS Hospital Anxiety and Depression scale
mTCNs Modified Toronto Clinical Neuropathy Scale
OSA Obstructive sleep apnoea
PSQI Pittsburgh Sleep Quality Index
RLS Restless leg syndrome 


\section{Introduction}

Patients with type 2 diabetes report sleep disturbances more frequently than individuals from the general population $[1,2]$. This is relevant for glucoregulation, as epidemiological [3-5] and experimental studies [6-8] have shown that reduced sleep duration and/or decreased sleep quality markedly reduce glucose tolerance and insulin sensitivity.

There are only a few studies on sleep characteristics in patients with type 1 diabetes.

Jauch-Chara et al. [9] reported a trend towards less slow wave sleep in 14 non-hypoglycaemic adult patients with type 1 diabetes. Children with type 1 diabetes had more disrupted sleep [10, 11] and more sleep disorders [12] than healthy children. Conversely, in adult patients with type 1 diabetes, partial sleep deprivation, even during only a single night, reduced peripheral insulin sensitivity by $21 \%$ [13]. Subjective sleep characteristics and their relation with glucoregulation have not been studied in adult patients with type 1 diabetes.

We hypothesised that adult patients with type 1 diabetes may have alterations in subjective sleep characteristics, assessed by validated sleep questionnaires, compared with healthy controls. In addition, we hypothesised that subjective sleep disturbances would be associated with impaired glucoregulation. Therefore, the aim of the present study was: (1) to assess subjective sleep characteristics by validated sleep questionnaires in adult patients with type 1 diabetes, compared with age-, sex- and BMI-matched non-diabetic controls; (2) to relate sleep characteristics to the quality of glycaemic control, i.e. $\mathrm{HbA}_{1 \mathrm{c}}$ values; and (3) to assess possible risk factors for impaired sleep characteristics in adult patients with type 1 diabetes.

\section{Methods}

\section{Participants}

We included 99 consecutive patients with type 1 diabetes mellitus (55 men, 44 women) attending the outpatient clinic of the Leiden University Medical Center, and 99 age-, sex- and BMI-matched non-diabetic controls recruited by advertisement. Every patient with type 1 diabetes was individually matched with one non-diabetic healthy control for age, sex and BMI.

Exclusion criteria for both groups were: (1) previously diagnosed sleep disorders; (2) psychiatric disorders and/or use of psychotropic medication; (3) pregnancy or lactation; (4) working in nights shifts in the last 3 months; (5) travelling across time zones in the previous month; (6) age $<18$ years; (7) other endocrine disorders; (8) neuropathy caused by other conditions than type 1 diabetes; (9) chronic co-morbidity, other than peripheral neuropathy, associated with pain; and (10) chronic use of glucocorticoids.

The study was approved by the medical ethical committee of Leiden University Medical Centre and written informed consent was obtained from all participants prior to the study.

\section{Study design}

Patients and controls were asked to complete three validated sleep questionnaires - the Pittsburgh Sleep Quality Index (PSQI), the Epworth Sleepiness Scale (ESS), and the Berlin Questionnaire (BQ) - that provide data on sleep quality, daytime sleepiness and the presence of sleeping disorders [14-16]. An additional questionnaire focused on duration and insulin management of type 1 diabetes, use of medication, co-morbidity, current smoking status and use of alcohol and coffee. Four questions aimed to identify restless legs syndrome (RLS), using the minimum criteria defined by the International RLS Study Group [17]. As depressive feelings may also affect sleep characteristics, anxiety and depression scores were assessed by the Hospital Anxiety and Depression scale (HADS) [18].

Sleep disturbances were identified by the answers of the ten possible encountered sleep disturbances of the PSQI questionnaire. The option 'three times or more per week' was taken as the affirmative answer. Habitual snoring was defined as a 'yes' answer to the three-item question 'Do you snore' in the BQ. The participants who answered 'not known' to this question were classified as non-snorers. We also included additional questions: 'Has your sleep been disturbed by hypoglycaemia in the past month? Has your sleep been disturbed by hyperglycaemia in the past month?' The following four options were provided: 'never'; 'less than once per week'; 'once or twice per week'; and 'three times or more per week'.

The following data were obtained from medical records for the 12 months preceding the current study. Microvascular complications were defined by the presence of: (1) retinopathy identified by retinal photography, detailed ophthalmologic examination and/or previous laser therapy; or (2) nephropathy identified by increased urinary albumin-to-creatinine ratios (men $>2.5 \mu \mathrm{g} / \mu \mathrm{mol}$, women $>3.5 \mu \mathrm{g} / \mu \mathrm{mol})$. Macrovascular complications were defined by objective documentation of coronary artery disease (diagnostic cardiac exercise test, coronary angiography, documented myocardial infarction and/or coronary artery bypass surgery or percutaneous coronary interventions), cerebral vascular disease (documented focal neurological findings supported by appropriate imaging studies) or peripheral vascular disease defined by reduced ankle-arm index or angiography. Hypertension was defined as systolic blood pressure $\geq 135 \mathrm{mmHg}$ or diastolic blood pressure $\geq 85 \mathrm{mmHg}$ at multiple 
occasions or treatment with antihypertensive medication. All patients had $\mathrm{HbA}_{1 \mathrm{c}}$ data obtained within the previous 3 months. An $\mathrm{HbA}_{1 \mathrm{c}}$ value of $7.5 \%$ (58 $\left.\mathrm{mmol} / \mathrm{mol}\right)$ was taken as the cut-off point dividing well-controlled vs moderate-to-poorly controlled patients.

The presence of peripheral polyneuropathy in the lower extremities was assessed by a single researcher (M. van Dijk), both in all type 1 diabetes patients and all controls, using the modified Toronto Clinical Neuropathy scale (mTCNs, see below) [19] and a neurothesiometer (Scientific Laboratory Supplies, Nottingham, UK). For the present study, peripheral polyneuropathy was considered to be present using a 2.5 percentile cut-off point for abnormality using data from the matched healthy controls of the present study, when either mTCNs score was $>5$ points or the vibration perception threshold was $>18.4 \mathrm{~V}$.

Assessment of subjective sleep characteristics

Pittsburgh Sleep Quality Index The validated PSQI is a 19-item self-rated questionnaire for assessing subjective sleep quality over the previous month. These questions generate seven 'component' scores, each weighted equally from 0 to 3: subjective sleep quality; sleep latency; sleep duration; habitual sleep efficiency; sleep disturbances; use of sleep medication; and daytime dysfunction. The global PSQI score ranges from 0 to 21 , with higher scores indicating worse sleep quality.

Epworth Sleepiness Scale The ESS is a validated eightitem questionnaire, focusing on the likelihood of falling asleep in several common situations encountered in daily life on a scale of 0 to 3 . Scores range from 0 (the least sleepy) to 24 (the most sleepy). Scores equal to, or above, ten are interpreted as hypersomnolence [15].

Berlin Questionnaire The BQ consists of ten questions on risk factors for sleep apnoea, subdivided into three symptom categories: (1) snoring and apnoeas (six points); (2) wake-time sleepiness or fatigue (three points); and (3) the presence of obesity or hypertension (two points). If two or more categories are positive (categories 1 and 2: two or more points; category 3: one or more points), a person is at high risk of having sleep apnoea [16].

Evaluation of depression

Hospital Anxiety and Depression Scale The HADS consists of 14 items scoring anxiety and depression. Each item is measured on a four-point scale. Score for anxiety and depression subscale range from 0 to 21 , and values of the total score range from 0 to 42 . Higher scores indicate more severe anxiety or depression. A total score of 13 or more was considered to be increased [18]. This questionnaire was provided to the participants after the initial study day. Of the 99 patients, $87(78.8 \%)$ and 80 of the 99 healthy controls $(80.8 \%)$ returned the HADS questionnaire. The other questionnaires were completed by all participating type 1 diabetic patients and controls.

Assessment of peripheral polyneuropathy

Modified Toronto Clinical Neuropathy Scale The mTCNs consists of a brief easily administered semi-structured clinical interview and clinical sensory examination to assess symptoms and signs of diabetic sensorimotor polyneuropathy. The mTCNS rates individual symptoms (foot pain, numbness, tingling, weakness, imbalance, upper limb symptoms) as absent or present, and if present, grades them depending on interference with sense of well-being or activities of daily living. Symptoms without interference with sense of well-being or activities of daily living are graded as 1 , those that interfere with sense of well-being, but not with activities of daily living as 2 , and those that interfere with both as 3 . The clinical sensory examination was performed unilaterally. In the case of previous trauma the unaffected leg was chosen, and otherwise the choice of the leg was random. Pinprick, temperature, light touch, vibration and position sense were graded as: (0) normal; (1) abnormal at the toes only; (2) between the toes and the ankle; or (3) or above the ankle [19]. For each of these modalities, reference stimuli were given to the dorsal site of one hand, after which testing was carried out on the sites described before, while individuals kept their eyes closed.

Neurothesiometer Before testing vibration perception threshold on the toes, participants were familiarised with vibration stimuli by applying these to the dorsum of one hand. Subsequently, vibration stimuli were applied bilaterally to the distal pulp of both toes. Individuals were instructed to indicate when vibration was first perceived. Vibration intensity was gradually increased until vibration was first detected by the patient or until a maximum output was reached. Testing was carried out with the individual's eyes closed. Testing was carried out twice on each toe, and a mean of both values was calculated in volts.

Statistical analysis

Data were analysed using PASW Statistics version 17.0.2 (SPSS, Chicago, IL, USA).

Continuous variables were described as mean \pm SEM; categorical variables were expressed as proportions. We used the paired $t$ test and the McNemar test for differences in means and proportions for continuous and categorical 
paired variables, respectively, and the two-tailed independent $t$ test and the $\chi^{2}$ test for unpaired data.

In patients with type 1 diabetes, logistic regression analysis was performed to examine the association of each sleep characteristic with poor glycaemic control (yes/no) adjusting for age, sex, BMI, use of alcohol (>1 glass/day, yes/no), and anxiety and depression scores according to the HADS.

To investigate variables for impaired sleep quality (PSQI>5) in the diabetic population, we fitted logistic regressions separately for all possible variables that could affect sleep quality: total exogenous insulin dose (units per kilogram per day), use of beta-blockers (yes/no), use of ACE inhibitors (yes/no), anxiety and depression score according to HADS, presence of hypertension (yes/no), nephropathy (yes/no), peripheral polyneuropathy (yes/no), macrovascular disease (yes/no), uncomfortable temperatures (yes/no), pain (yes/no), polyuria (yes/no), other sleep disturbances (e.g. metabolic dysregulation), habitual snoring (yes/no), high risk for OSA (yes/no), and RLS (yes/no). In these analyses, we adjusted for the confounders age, sex and BMI by including them as covariates. Subsequently, we performed multivariate logistic regression analysisincluding age, sex, BMI, $\mathrm{HbA}_{1 \mathrm{c}}$, duration of the diabetes and the risk factors that showed a $p$ value of $<0.2$ in the preceding separate analyses.

\section{Results}

\section{Clinical characteristics}

We included 99 patients with type 1 diabetes and 99 healthy non-diabetic controls matched for sex (55 men, 44 women), age $(43.9 \pm 1.3$ vs $44.1 \pm 1.3$ years $)$ and BMI $(24.5 \pm 0.3$ vs $24.5 \pm 0.3 \mathrm{~kg} / \mathrm{m}^{2}$ ). The two groups did not differ with respect to current smoking status or use of alcohol or coffee. Clinical characteristics are summarised in Table 1.

Patients with type 1 diabetes used more frequently ACE inhibitors, calcium antagonists, statins, angiotensin II receptor antagonists and anti-platelet agents. According to the HADS, both anxiety (5.0 \pm 0.4 vs $3.7 \pm 0.3, p=0.004$ ) and depression scores $(3.3 \pm 0.4$ vs $1.6 \pm 0.2, p=0.001)$ were significantly higher in the patients with type 1 diabetes. Thirteen patients (13.1\%) had elevated scores for anxiety and depression (total HADS score 13 or more) vs six $(6.1 \%)$ of the controls $(p=0.267)$. The mean duration of the diabetes was $26.9 \pm 1.2$ years. $\mathrm{HbA}_{1 \mathrm{c}}$ values were $7.8 \pm$ $0.1 \%(62 \pm 1.3 \mathrm{mmol} / \mathrm{mol})$. Multiple daily insulin injections were used by 85 patients and the other 14 patients used continuous s.c. insulin infusion. The mean insulin dose was $0.70 \pm 0.0 \mathrm{Ukg}^{-1} \mathrm{day}^{-1}$. Microvascular complications were present in 36 patients: 24 patients had retinopathy and 19 patients had nephropathy. The criteria for peripheral polyneuropathy were met by 45 patients compared with two of the matched healthy controls $(p=0.000)$. Peripheral polyneuropathy was considered to be present, using a 2.5 percentile cut-off point for abnormality using data from the matched healthy controls of the present study, when either mTCNs score was $>5$ points or the vibration perception threshold was $>18.4 \mathrm{~V}$. Macrovascular complications were present in 15 patients.

Subjective sleep characteristics Self-reported mean duration of sleep in the PSQI did not differ between patients and controls (7.2 \pm 0.1 vs $7.1 \pm 0.1 \mathrm{~h}, p=0.372$; Table 2). In addition, the proportion of short sleepers $(<5 \mathrm{~h})$ did not differ significantly between the two groups ( $1 \%$ vs $1 \%, p=$ 1.00 ), and nor did the proportion of long sleepers ( $>9 \mathrm{~h}: 1 \%$ vs $1 \%, p=1.00$ ). Although there were no significant differences in global PSQI scores between patients and controls, $35.4 \%$ of the patients had a total PSQI score $>5$, compared with only $19.2 \%$ of the controls, indicating a higher number of patients with poor sleep quality $(p=0.021)$. In the individual domains of the PSQI questionnaire, patients more often reported sleep disturbances $(p=0.001)$ and daytime dysfunction $(p=0.031)$. The following sleep disturbances occurred more often in patients than in controls: polyuria ( $42.4 \%$ vs $24.2 \%, p=0.004)$, pain $(8.1 \%$ vs $0 \%, p=0.008)$, uncomfortable temperatures $(8.1 \%$ vs $1.0 \%, p=0.021)$ and habitual snoring ( $51 \%$ vs $34 \%, p=0.010)$.

The BQ indicated that more patients were at high risk for obstructive sleep apnoea (OSA) than controls (17.2 vs $5.1 \%, p=0.012)$. Despite the reduction in sleep quality and more sleep disorders, there were no significant differences in reported daytime sleepiness between the two groups. Daytime hypersomnolence affected $19.2 \%$ of the patients vs $11.1 \%$ of the controls $(p=0.152)$.

Seventy-five patients answered the questions: 'Has your sleep been disturbed by hypoglycaemia in the past month? Has your sleep been disturbed by hyperglycaemia in the past month?' Forty-one patients indicated that their sleep was disturbed by hypoglycaemia in the past month: 34 patients indicated that their sleep was disturbed less than once per week, and seven patients indicated impaired sleep by hypoglycaemia one to two times per week. Disturbed sleep due to hyperglycaemia was reported by 28 patients with type 1 diabetes: 24 patients indicated disturbed sleep less than once per week, two patients one to two times per week, and two patients reported a disturbed sleep by hyperglycaemia more than three times per week.

Well-controlled vs moderate-to-poorly controlled patients

Type 1 diabetes patients were divided in two groups: well controlled $\left(\mathrm{HbA}_{1 \mathrm{c}}<7.5 \% \quad[<58 \mathrm{mmol} / \mathrm{mol}], n=51\right)$ vs 
Table 1 Clinical characteristics of the patients with type 1 diabetes and their non-diabetic matched controls
Data are mean \pm SEM for continuous variables and $n(\%)$ for categorical variables

NA, not applicable
Patients with type 1 diabetes $(n=99)$

Sex

Male/female
Age (years)
BMI $\left(\mathrm{kg} / \mathrm{m}^{2}\right)$
$\mathrm{BMI} \geq 30 \mathrm{~kg} / \mathrm{m}^{2}$
$\mathrm{HbA}_{1 \mathrm{c}}$
$\%$
$\mathrm{mmol}^{2} \mathrm{~mol}$
$\mathrm{HbA}_{1 \mathrm{c}} \geq 7.5 \%(\geq 58 \mathrm{mmol} / \mathrm{mol})$

Duration of diabetes (years)

Mean insulin dose $\left(\mathrm{U} \mathrm{kg}^{-1} \mathrm{day}^{-1}\right)$

Medications, $n$ (\%)

Insulin

Continuous s.c. insulin infusion

Insulin injections

Beta-blockers

ACE inhibitors

Calcium antagonists

Statins

Oral glucose-lowering agents

Angiotensin II receptor antagonists

Diuretics

Oral contraceptives

Anti-platelet agents

Proton pump inhibitors

Benzodiazepines

Other

Diabetic complications, $n(\%)$

Microvascular

Retinopathy

Laser treatment

Nephropathy

Macrovascular

Peripheral polyneuropathy

mTCNs score

Neurothesiometer (V)

Hypertension, $n(\%)$

Current cigarette smokers, $n(\%)$

Alcohol consumption $\geq 1$ glass/day, $n(\%)$

Coffee consumption $\geq 1$ cup/day, $n(\%)$

HADS

$\begin{array}{ll}\text { Anxiety score } & 5.0 \pm 0.4 \\ \text { Depression score } & 3.3 \pm 0.4 \\ \text { Total score } & 8.3 \pm 0.7 \\ \text { Score } \geq 13 & 13(13.1)\end{array}$

Controls $(n=99) \quad p$ value

$55 / 44$

$55 / 44$

1.000

$43.9 \pm 1.3$

$44.1 \pm 1.3$

0.472

$24.5 \pm 0.3$

$24.5 \pm 0.3$

0.986

$4(4.0)$

$6(6.1)$

0.727

NA

$7.8 \pm 0.1$

$62 \pm 1.3$

48 (48.5)

$26.9 \pm 1.2$

NA

$0.7 \pm 0.0$

NA

NA

$14(14.0)$

$86(86.0)$

$6(6.1)$

$4(4.0)$

0.754

$29(29.3)$

$9(9.1)$

39 (39.4)

$1(1.0)$

$9(9.1)$

$8(8.1)$

$7(7.1)$

17 (17.2)

$5(5.1)$

0.000

$2(2.0) \quad 0.039$

$6(6.1) \quad 0.000$

0

$1(1.0)$

1.000

$1(1.0)$

0.021

$3(3.0)$

0.227

$8(8.1)$

1.000

2 (2.0)

0.000

$6(6.1)$

$2(2.0)$

0.289

$24(24.2)$

0

18 (18.2) $\quad 0.391$

NA

$36(36.4)$

$24(24.2)$

18 (18.2)

19 (19.2)

15 (15.0)

45 (45.5)

$2(2.0)$

0.000

$5.6 \pm 0.5$

$1.4 \pm 0.2$

0.000

$11.5 \pm 0.9$

$6.8 \pm 0.4$

0.000

24 (22.0)

18 (18.0)

11 (11.0)

0.210

41 (41.4)

36 (36.4)

0.551

79(79.8)

0.307

75 (75.8)

$3.7 \pm 0.3$

0.004

$1.6 \pm 0.2$

0.001

Score $\geq 13$

$5.3 \pm 0.5$

0.002

$6(6.1)$

0.388 moderate to poorly controlled $\left(\mathrm{HbA}_{1 \mathrm{c}} \geq 7.5 \%\right.$ [ $\left.\geq 58 \mathrm{mmol} / \mathrm{mol}\right]$, $n=48$ ). There were no significant differences in clinical characteristics between both groups. We found no significant differences between both groups in subjective sleep characteristics, i.e. self-reported sleep duration, sleep quality, daytime sleepiness, or the prevalence of 
Table 2 Subjective sleep characteristics of the patients with type 1 diabetes vs matched non-diabetic controls
Data are mean \pm SEM for continuous variables and $n(\%)$ for categorical variables

\begin{tabular}{|c|c|c|c|}
\hline Sleep characteristic & Patients with type 1 diabetes $(n=99)$ & Controls $(n=99)$ & $p$ value \\
\hline \multicolumn{4}{|l|}{ Sleep duration } \\
\hline Self-reported sleep duration (h) & $7.2 \pm 0.1$ & $7.1 \pm 0.1$ & 0.372 \\
\hline \multicolumn{4}{|l|}{ Sleep quality } \\
\hline \multicolumn{4}{|l|}{ PSQI } \\
\hline Global score & $4.6 \pm 0.3$ & $4.0 \pm 0.2$ & 0.079 \\
\hline PSQI $>5, n(\%)$ & $36(35.4)$ & $20(19.8)$ & 0.021 \\
\hline \multicolumn{4}{|l|}{ Component score } \\
\hline 1. Subjective sleep quality & $0.88 \pm 0.1$ & $0.73 \pm 0.1$ & 0.096 \\
\hline 2. Sleep latency & $0.68 \pm 0.1$ & $0.74 \pm 0.1$ & 0.598 \\
\hline 3. Sleep duration & $0.73 \pm 0.1$ & $0.77 \pm 0.1$ & 0.781 \\
\hline 4. Sleep efficiency & $0.31 \pm 0.1$ & $0.22 \pm 0.1$ & 0.307 \\
\hline 5. Sleep disturbances & $1.12 \pm 0.0$ & $0.92 \pm 0.0$ & 0.002 \\
\hline 6. Sleeping medication & $0.13 \pm 0.0$ & $0.06 \pm 0.0$ & 0.239 \\
\hline 7. Daytime dysfunction & $0.75 \pm 0.1$ & $0.52 \pm 0.1$ & 0.031 \\
\hline \multicolumn{4}{|l|}{ Daytime sleepiness } \\
\hline \multicolumn{4}{|l|}{ ESS } \\
\hline Total score & $5.9 \pm 0.4$ & $5.1 \pm 0.4$ & 0.192 \\
\hline Score $\geq 10, n(\%)$ & $19(19.2)$ & $11(11.1)$ & 0.152 \\
\hline \multicolumn{4}{|l|}{ Sleep disorders, $n(\%)$} \\
\hline \multicolumn{4}{|l|}{ BQ } \\
\hline Habitual snoring & $51(51.0)$ & $34(34.0)$ & 0.010 \\
\hline High-risk OSA & $17(17.2)$ & $5(5.1)$ & 0.012 \\
\hline RLS & $9(9.3)$ & $10(10.1)$ & 1.000 \\
\hline
\end{tabular}

sleep disorders (data not shown). Logistic regression analysis was performed to assess the association between self-reported sleep characteristics and high $\mathrm{HbA}_{1 \mathrm{c}}$ values $(\geq 7.5 \% \quad[\geq 58 \mathrm{mmol} / \mathrm{mol}])$. There were no significant associations between individual sleep characteristics and impaired glycaemic control (Table 3).

Assessment of risk factors of impaired sleep quality (PSQI $>5$ ) in patients with type 1 diabetes

Logistic regression analyses for all possible variables that could affect sleep quality in patients with type 1 diabetes showed significant associations between impaired sleep quality and the presence of peripheral polyneuropathy (OR 2.98, 95\% CI 1.05-8.46, $p=0.040$ ), habitual snoring (OR 4.19, 95\% CI 1.47-11.97, $p=0.007$ ), uncomfortable temperatures (OR 7.89, 95\% CI 1.31-47.31, $p=0.024$ ), other sleep disturbances, e.g. metabolic dysregulation (OR 9.52, 95\% CI $1.03-88.17, p=0.047)$, HADS anxiety score (OR 1.20, 95\% CI 1.02-1.40, $p=0.026$ ), and HADS depression score (OR 1.30, 95\% CI 1.07-1.58, $p=0.009$; Table 4).

Multivariate logistic regression, including age, sex, BMI, $\mathrm{HbA}_{1 \mathrm{c}}$, duration of diabetes and the risk factors, that showed a $p$ value $<0.2$ in the preceding separate analyses confirmed that habitual snoring (OR 9.95, 95\% CI 1.76-
Table 3 Results of logistic regression analysis of the association between sleep characteristics and impaired glucoregulation in 99 patients with type 1 diabetes

\begin{tabular}{llc}
\hline Sleep characteristic & $\mathrm{HbA}_{1 \mathrm{c}} \geq 7.5 \%(\geq 58 \mathrm{mmol} / \mathrm{mol})$ \\
\cline { 2 - 3 } & $\mathrm{OR}(95 \% \mathrm{CI})$ & $p$ value \\
\hline Sleep duration $^{\mathrm{a}}$ & & \\
$\quad$ Self-reported sleep duration (h) & $0.77(0.48-1.24)$ & 0.287 \\
Sleep quality & & \\
PSQI & & \\
$\quad$ Global PSQI score & $1.06(0.89-1.27)$ & 0.532 \\
$\quad$ PSQI $>5$ & $1.31(0.47-3.63)$ & 0.602 \\
Daytime sleepiness & \\
ESS & & \\
Total ESS-score & $1.04(0.92-1.18)$ & 0.521 \\
ESS $\geq 10$ & $2.82(0.84-9.42)$ & 0.093 \\
Sleep disorders & & \\
BQ & & 0.639 \\
Habitual snoring & $1.24(0.51-3.01)$ & 0.238 \\
High-risk OSA & $0.50(0.15-1.59)$ & 0.209 \\
RLS & $2.59(0.59-11.41)$ \\
\hline
\end{tabular}

Each sleep characteristic was adjusted for age, sex, BMI and alcohol consumption ( $>1$ glass/day)

${ }^{\mathrm{a}}$ Also adjusted for HADS 
Table 4 Results of logistic regression: possible risk factors for impaired sleep quality (PSQI $>5$ ) in 99 patients with type 1 diabetes

\begin{tabular}{|c|c|c|c|c|}
\hline \multirow[t]{3}{*}{ Characteristic } & \multicolumn{4}{|c|}{ Poor sleep quality (PSQI >5) } \\
\hline & \multicolumn{2}{|c|}{$\begin{array}{l}\text { Minimally adjusted model } \\
\text { (adjusted for age, sex and BMI) }\end{array}$} & \multicolumn{2}{|c|}{ Maximally adjusted model } \\
\hline & OR $(95 \% \mathrm{CI})$ & $p$ value & OR $(95 \% \mathrm{CI})$ & $p$ value \\
\hline \multicolumn{5}{|l|}{ Clinical characteristics } \\
\hline \multicolumn{5}{|l|}{ Use of medication } \\
\hline Total insulin dose $\left(\mathrm{U} \mathrm{kg}^{-1}\right.$ day $\left.^{-1}\right)$ & $1.32(0.21-8.31)$ & 0.768 & $\mathrm{NI}$ & \\
\hline Use of beta-blockers & $1.00(0.17-5.92)$ & 0.996 & NI & \\
\hline Use of ACE inhibitors & $0.42(0.14-1.25)$ & 0.118 & $0.85(0.14-5.11)$ & 0.859 \\
\hline \multicolumn{5}{|l|}{ Anxiety and depressive symptoms } \\
\hline HADS anxiety score & $1.20(1.02-1.40)$ & 0.026 & $0.91(0.66-1.26)$ & 0.572 \\
\hline HADS depression score & $1.30(1.07-1.58)$ & 0.009 & $1.42(1.00-2.02)$ & 0.048 \\
\hline \multicolumn{5}{|l|}{ Diabetic complications } \\
\hline Hypertension & $0.41(0.13-1.31)$ & 0.133 & $0.38(0.06-2.32)$ & 0.292 \\
\hline Presence of nephropathy & $0.88(0.29-2.68)$ & 0.828 & NI & \\
\hline Macrovascular disease & $0.78(0.21-2.92)$ & 0.707 & NI & \\
\hline Presence of peripheral neuropathy & $3.0(1.05-8.46)$ & 0.040 & $7.45(1.08-51.20)$ & 0.041 \\
\hline \multicolumn{5}{|l|}{ Sleep disturbances } \\
\hline Uncomfortable temperatures & $7.89(1.31-47.31)$ & 0.024 & $4.30(0.27-69.45)$ & \\
\hline Presence of pain & $3.38(0.72-15.84)$ & 0.122 & $6.50(0.50-84.43)$ & 0.152 \\
\hline Polyuria & $1.49(0.20-3.97)$ & 0.405 & NI & \\
\hline Other sleep disturbances, (e.g. metabolic dysregulation) & $9.52(1.03-88.17)$ & 0.047 & $27.78(1.11-697.48)$ & 0.043 \\
\hline \multicolumn{5}{|l|}{ Sleep disorders } \\
\hline Habitual snoring & $4.20(1.47-11.97)$ & 0.007 & $9.95(1.76-56.14)$ & 0.009 \\
\hline High-risk OSA & $1.27(0.40-4.05)$ & 0.682 & NI & \\
\hline RLS & $0.89(0.20-3.97)$ & 0.883 & NI & \\
\hline
\end{tabular}

NI, not included in final model because $p>0.2$

56.14, $p=0.009$ ), HADS depression score (OR 1.42, 95\% CI $1.00-2.02, p=0.048)$, presence of peripheral polyneuropathy (OR 7.45, 95\% CI 1.08-51.20, $p=0.041$ ) and other sleep disturbances (e.g. metabolic dysregulation [OR 27.78, 95\% CI 1.11-697.48, $p=0.043]$ ) were still independent risk factors for poor sleep in the type 1 diabetes patients. Peripheral polyneuropathy, clinically assessed according to strict criteria, was an independent risk factor for impaired sleep, even after adjusting for 'pain' and 'uncomfortable temperatures'.

\section{Discussion}

The aim of this study was to assess subjective sleep characteristics in adult patients with long-standing type 1 diabetes, and to relate sleep variables to $\mathrm{HbA}_{1 \mathrm{c}}$ values. Although sleep duration did not differ between patients and controls, more patients had poor sleep quality compared with non-diabetic, age-, sex- and BMI-matched controls. Patients with type 1 diabetes reported more sleep disturbances and daytime dysfunction. A higher proportion of the patients with type 1 diabetes were at increased risk for OSA. There was no association between subjective sleep characteristics and impaired glucoregulation. These observations indicate that type 1 diabetes is associated with an increased prevalence of disturbed subjective sleep characteristics, which do not relate to glucoregulation.

Previous studies on the relation between diabetes and sleep characteristics mainly focussed on patients with type 2 diabetes [1,2]. Only a few studies have assessed sleep characteristics in patients with type 1 diabetes [9-12]. Those studies investigated relatively few individuals and children [10-12] with type 1 diabetes. The present study extends those observations in showing that in a large group of adult patients with a long history of type 1 diabetes subjective sleep characteristics are impaired, compared with a carefully matched control group, controlling for potential confounding factors such as age, sex and BMI.

This decrease in sleep quality and increased prevalence of sleep disturbances in patients with long-standing type 1 diabetes may have important implications, as previous studies showed that reduction of sleep duration and/or 
decreased sleep quality impair glucose tolerance and reduce insulin sensitivity in healthy controls [6-8]. Sleep disturbances might have a similar negative effect on glucose metabolism in patients with type 1 diabetes, resulting in worse diabetic control. However, this presumed relationship between sleep disturbances and impaired glucose metabolism, assessed by $\mathrm{HbA}_{1 \mathrm{c}}$ values, was not detectable in the current study. Nonetheless, it is still possible that disturbed sleep characteristics influence glucose metabolism in these patients. However, the effects of impaired sleep characteristics may not simply be reflected in $\mathrm{HbA}_{1 \mathrm{c}}$ values because intensive glucose control and frequent, appropriate adjustments of insulin doses in patients at risk might have obtunded the effects of impaired sleep characteristics on glucoregulation.

Various aspects of diabetes could be linked to disturbed sleep quality, including physical complications of the disease, psychological factors, metabolic fluctuations and high prevalence of sleep disorders. In the patients with type 1 diabetes in our study, disturbed sleep quality was independently associated with habitual snoring, higher depression scores according to the HADS questionnaire, presence of polyneuropathy and other sleep disturbances, mainly by hypoglycaemia.

Previous studies showed a high prevalence of depression in diabetes [20] and chronic pain conditions [21]. Although we excluded patients with a known depression, use of psychotropic drugs, and co-morbid disorders (other than neuropathy) associated with pain, in our study higher depression scores were independently associated with impaired sleep quality.

Many patients with type 1 diabetes in our study used ACE inhibitors, statins and/or beta-blockers, which might interfere with sleep characteristics. The effects of betablockers on sleep are not equivocal. A previous study showed that the use of beta-blockers could positively or negatively affect sleep [22]. A case report by Cicolin et al. suggested that ACE inhibitors may contribute to OSA by inducing upper airway inflammation [23]. Therefore, we have considered that the use of beta-blockers and/or ACE inhibitors might affect sleep quality in patients with type 1 diabetes. However, in univariate logistic regression analysis we did not find an association between the use of betablockers or ACE inhibitors and impaired sleep quality. In univariate logistic regression analysis there was an association between the use of ACE inhibitors and high risk of OSA. However, this association was no longer significant after correction for the confounders age, sex, BMI and hypertension. There are conflicting data on sleep disturbances in patients treated with statins. Some studies reported higher prevalence of sleep disturbances in patients treated with lipophilic statins than with pravastatin $[24,25]$ whereas other studies did not find an increased prevalence of sleep disturbances in patients treated with different statins compared with placebo [26, 27]. In the present study, there was no difference in the use of statins between patients with a poor sleep quality $(\mathrm{PSQI}>5)$ and patients with a good sleep quality (PSQI $\leq 5)$. The use of statins was even higher in the group with good sleep quality. In accordance, in univariate analysis, use of statins was not associated with impaired sleep quality. Therefore, our conclusions are not likely to be merely explained by the use of medications in our patients.

The clinical assessment of peripheral polyneuropathy according to strict criteria in individuals with type 1 diabetes is a major strength of our study, as our study shows diabetic polyneuropathy was a major determinant of impaired sleep. Polyneuropathy contributes to impaired sleep via several potential mechanisms. First, neuropathic pain may lead to disturbed sleep [28]. Second, polyneuropathy can impair thermoregulation. It has been proposed that autonomic changes in skin temperature modulate the neuronal activity of the thermosensitive neurons in the pre-optic area/anterior hypothalamus, which, in turn, regulate vigilance and sleepiness [29]. This hypothesis is supported by a report showing that diabetic patients, even those without evidence of clinical neuropathy, show impaired thermoregulation during sleep [30].

The relatively high prevalence of type 1 diabetic patients with a high risk for OSA, according to the BQ, suggests the potential of a high burden of unrecognised OSA in people with type 1 diabetes. This is a relatively new finding, in accordance with a recent pilot study of Borel et al., which observed a prevalence of OSA of $40 \%$ in 37 non-obese adult patients with type 1 diabetes [31]. In accordance with our data, this observation is remarkable, as the BMI, which is a risk factor for OSA in the general population, of our patients with type 1 diabetes was matched to that of the healthy controls. Several studies in patients with type 2 diabetes have shown that OSA is associated with the presence of autonomic neuropathy $[32,33]$, which might also be involved in patients with type 1 diabetes. Unfortunately, our current study was not designed to elucidate underlying mechanisms of disturbed sleep, and we did not include assessments of autonomic neuropathy. Nonetheless, there was no association between poor glycaemic control $\left(\mathrm{HbA}_{1 \mathrm{c}} \geq\right.$ $7.5 \%$ ) and a 'high' risk for OSA in our study, despite the association between sleep-disordered breathing, glucose intolerance and insulin resistance in patients with type 2 diabetes $[34,35]$. There was also no association between the occurrence of hypoglycaemia and the risk for OSA in our patients [36].

Sleep characteristics were assessed by validated questionnaires in the present study. The PSQI and ESS 
have been developed to measure sleep quality and daytime sleepiness, respectively [14, 15], and reflect stable measures of sleep quality and sleepiness over the past year [37]. The PSQI has a diagnostic sensitivity of $89.6 \%$ and a specificity of $86.5 \%$ for identifying cases with poor sleep quality, using a cut-off score of 5 . This questionnaire has been validated by polysomnographic measurements [38].The BQ is a screening tool widely used to differentiate between 'high-' and 'low-risk' groups for OSA. This risk grouping was useful in the prediction of respiratory disturbances in consecutive participants, who visited internists for any reason. For example, being in the 'high-risk' group defined by the BQ predicted more than five respiratory events per hour with a sensitivity of $86 \%$, and a specificity of $77 \%$ [16]. In view of the current data, polysomnography is required to objectively assess the sleep quality and OSA in patients with type 1 diabetes mellitus at high risk for OSA according to the $\mathrm{BQ}$.

The current cross-sectional study was designed to assess subjective sleep variables in patients with type 1 diabetes mellitus and, therefore, we cannot elucidate from the data which chain(s) of events lead from type 1 diabetes to disturbed sleep. In particular, the links between peripheral polyneuropathy and disturbed sleep and between type 1 diabetes and the risk of OSA are not fully clear. Another matter is whether disturbed sleep leads to further impairment of glucose metabolism, with the effect that sleep disturbances and glycaemic control can interact in a vicious circle. Additional studies with objective sleep measurements are warranted to assess these relations in more detail.

In conclusion, the present study demonstrated that adult patients with long-standing type 1 diabetes mellitus have altered self-reported sleep characteristics compared with sex-, age- and BMI-matched non-diabetic controls. Therefore, disturbed subjective sleep characteristics are part of the complex syndrome of long-standing type 1 diabetes mellitus.

\begin{abstract}
Acknowledgements We gratefully acknowledge support for this study from the Clinical Research Grant from the European Foundation for the Study of Diabetes (EFSD). We thank all the volunteers for participating in this study. The authors also thank E. W. van Zwet of the Department of Medical Statistics, Leiden University Medical Centre, for help with the statistical analyses.
\end{abstract}

Duality of interest The authors declare that there is no duality of interest associated with this manuscript.

Open Access This article is distributed under the terms of the Creative Commons Attribution Noncommercial License which permits any noncommercial use, distribution, and reproduction in any medium, provided the original author(s) and source are credited.

\section{References}

1. Skomro RP, Ludwig S, Salamon E, Kryger MH (2001) Sleep complaints and restless legs syndrome in adult type 2 diabetics. Sleep Med 2:417-422

2. Resnick HE, Redline S, Shahar E et al (2003) Diabetes and sleep disturbances: findings from the Sleep Heart Health Study. Diabetes Care 26:702-709

3. Gottlieb DJ, Punjabi NM, Newman AB et al (2005) Association of sleep time with diabetes mellitus and impaired glucose tolerance. Arch Intern Med 165:863-867

4. Ayas NT, White DP, Al-Delaimy WK et al (2003) A prospective study of self-reported sleep duration and incident diabetes in women. Diabetes Care 26:380-384

5. Yaggi HK, Araujo AB, McKinlay JB (2006) Sleep duration as a risk factor for the development of type 2 diabetes. Diabetes Care 29:657-661

6. Spiegel K, Leproult R, van CE (1999) Impact of sleep debt on metabolic and endocrine function. Lancet 354:1435-1439

7. Donga E, van Dijk M, van Dijk JG et al (2010) A single night of partial sleep deprivation induces insulin resistance in multiple metabolic pathways in healthy subjects. J Clin Endocrinol Metab 95:2963-2968

8. Tasali E, Leproult R, Ehrmann DA, van CE (2008) Slow-wave sleep and the risk of type 2 diabetes in humans. Proc Natl Acad Sci USA 105:1044-1049

9. Jauch-Chara K, Schmid SM, Hallschmid M, Born J, Schultes B (2008) Altered neuroendocrine sleep architecture in patients with type 1 diabetes. Diabetes Care 31:1183-1188

10. Happe S, Treptau N, Ziegler R, Harms E (2005) Restless legs syndrome and sleep problems in children and adolescents with insulin-dependent diabetes mellitus type 1. Neuropediatrics 36:98-103

11. Matyka KA, Crawford C, Wiggs L, Dunger DB, Stores G (2000) Alterations in sleep physiology in young children with insulindependent diabetes mellitus: relationship to nocturnal hypoglycemia. J Pediatr 137:233-238

12. Villa MP, Multari G, Montesano M et al (2000) Sleep apnoea in children with diabetes mellitus: effect of glycaemic control. Diabetologia 43:696-702

13. Donga E, van Dijk M, van Dijk JG et al (2010) Partial sleep restriction decreases insulin sensitivity in type 1 diabetes. Diabetes Care 33:1573-1577

14. Buysse DJ, Reynolds CF III, Monk TH, Berman SR, Kupfer DJ (1989) The Pittsburgh Sleep Quality Index: a new instrument for psychiatric practice and research. Psychiatry Res 28:193-213

15. Johns MW (1991) A new method for measuring daytime sleepiness: the Epworth sleepiness scale. Sleep 14:540-545

16. Netzer NC, Stoohs RA, Netzer CM, Clark K, Strohl KP (1999) Using the Berlin Questionnaire to identify patients at risk for the sleep apnea syndrome. Ann Intern Med 131:485-491

17. Allen RP, Picchietti D, Hening WA, Trenkwalder C, Walters AS, Montplaisi J (2003) Restless legs syndrome: diagnostic criteria, special considerations, and epidemiology. A report from the Restless Legs Syndrome Diagnosis and Epidemiology Workshop at the National Institutes of Health. Sleep Med 4:101-119

18. Zigmond AS, Snaith RP (1983) The hospital anxiety and depression scale. Acta Psychiatr Scand 67:361-370

19. Bril V, Tomioka S, Buchanan RA, Perkins BA (2009) Reliability and validity of the modified Toronto Clinical Neuropathy Score in diabetic sensorimotor polyneuropathy. Diabet Med 26:240-246

20. Anderson RJ, Freedland KE, Clouse RE, Lustman PJ (2001) The prevalence of comorbid depression in adults with diabetes: a meta-analysis. Diabetes Care 24:1069-1078 
21. Gore M, Brandenburg NA, Dukes E, Hoffman DL, Tai KS, Stacey B (2005) Pain severity in diabetic peripheral neuropathy is associated with patient functioning, symptom levels of anxiety and depression, and sleep. J Pain Symptom Manage 30:374-385

22. Yilmaz MB, Erdem A, Yalta K, Turgut OO, Yilmaz A, Tandogan I (2008) Impact of beta-blockers on sleep in patients with mild hypertension: a randomized trial between nebivolol and metoprolol. Adv Ther 25:871-883

23. Cicolin A, Mangiardi L, Mutani R, Bucca C (2006) Angiotensinconverting enzyme inhibitors and obstructive sleep apnea. Mayo Clin Proc 81:53-55

24. Golomb BA, Kwon EK CMDJ (2011) Abstract 3725: Simvastatin but not pravastatin affects sleep: findings from the UCSD Statin Study. Circulation 116:II 847 (Abstract)

25. Vgontzas AN, Kales A, Bixler EO, Manfredi RL, Tyson KL (1991) Effects of lovastatin and pravastatin on sleep efficiency and sleep stages. Clin Pharmacol Ther 50:730-737

26. Eckernas SA, Roos BE, Kvidal P et al (1993) The effects of simvastatin and pravastatin on objective and subjective measures of nocturnal sleep: a comparison of two structurally different HMG CoA reductase inhibitors in patients with primary moderate hypercholesterolaemia. Br J Clin Pharmacol 35:284-289

27. Ehrenberg BL, Lamon-Fava S, Corbett KE, McNamara JR, Dallal GE, Schaefer EJ (1999) Comparison of the effects of pravastatin and lovastatin on sleep disturbance in hypercholesterolemic subjects. Sleep 22:117-121

28. Zelman DC, Brandenburg NA, Gore M (2006) Sleep impairment in patients with painful diabetic peripheral neuropathy. Clin J Pain 22:681-685

29. Raymann RJ, Swaab DF, van Someren EJ (2008) Skin deep: enhanced sleep depth by cutaneous temperature manipulation. Brain 131:500-513
30. Rutkove SB, Veves A, Mitsa T et al (2009) Impaired distal thermoregulation in diabetes and diabetic polyneuropathy. Diabetes Care 32:671-676

31. Borel AL, Benhamou PY, Baguet JP et al (2010) High prevalence of obstructive sleep apnoea syndrome in a type 1 diabetic adult population: a pilot study. Diabet Med 27:1328-1329

32. Ficker JH, Dertinger SH, Siegfried W et al (1998) Obstructive sleep apnoea and diabetes mellitus: the role of cardiovascular autonomic neuropathy. Eur Respir J 11:14-19

33. Bottini P, Dottorini ML, Cristina CM, Casucci G, Tantucci C (2003) Sleep-disordered breathing in nonobese diabetic subjects with autonomic neuropathy. Eur Respir J 22:654-660

34. Harsch IA, Schahin SP, Bruckner K et al (2004) The effect of continuous positive airway pressure treatment on insulin sensitivity in patients with obstructive sleep apnoea syndrome and type 2 diabetes. Respiration 71:252-259

35. Punjabi NM, Shahar E, Redline S, Gottlieb DJ, Givelber R, Resnick HE (2004) Sleep-disordered breathing, glucose intolerance, and insulin resistance: the Sleep Heart Health Study. Am J Epidemiol 160:521-530

36. Paranjape SA, Vavaiya KK, Kale AY, Briski KP (2006) Habituation of insulin-induced hypoglycemic transcription activation of lateral hypothalamic orexin-A-containing neurons to recurring exposure. Regul Pept 135:1-6

37. Knutson KL, Rathouz PJ, Yan LL, Liu K, Lauderdale DS (2006) Stability of the Pittsburgh Sleep Quality Index and the Epworth Sleepiness Questionnaires over 1 year in early middle-aged adults: the CARDIA Study. Sleep 29:1503-1506

38. Buysse DJ, Reynolds CF III, Monk TH, Hoch CC, Yeager AL, Kupfer DJ (1991) Quantification of subjective sleep quality in healthy elderly men and women using the Pittsburgh Sleep Quality Index (PSQI). Sleep 14:331-338 